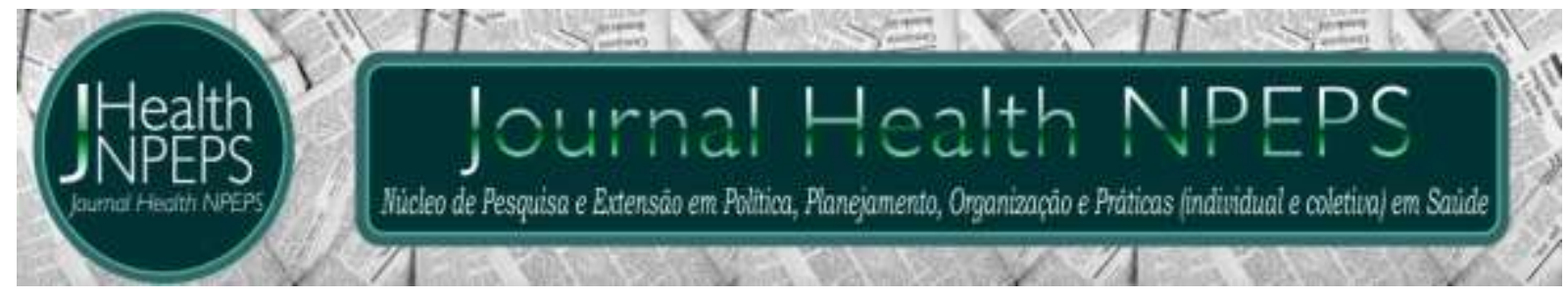

http://dx.doi.org/10.30681/252610103981

ARTIGO ORIGINAL

\title{
Prevenção de lesão por pressão: conhecimento e ações de cuidadores e pacientes domiciliares
}

\section{Pressure ulcer prevention: knowledge and actions of caregivers and home patients}

\section{Prevención de lesion por presión: conocimiento y acciones de cuidadores y pacientes domiciliarios}

\section{Tatyelle Bezerra Carvalho1, Luis Rafael Leite Sampaio², Brenda Belém Luna Sampaio ${ }^{3}$, Maria Neyze Martins Fernandes ${ }^{4}$, Cleide Correia de Oliveira ${ }^{5}$}

\section{RESUMO}

Objetivo: conhecer as ações realizadas no domicílio para a prevenção de lesão por pressão na Estratégia Saúde da Família. Método: pesquisa descritiva e qualitativa, direcionada à população em risco para o desenvolvimento de lesão por pressão (LP) no município de Crato, Ceará, Brasil. A coleta de dados foi realizada por intermédio da escala de Braden e entrevista semiestruturada, nos meses de março a maio de 2018. Os aspectos éticos envolvidos na pesquisa foram respeitados. Resultados: os resultados obtidos foram divididos em duas categorias: 1) análise do risco de desenvolvimento de LP a partir da escala de Braden; 2) percepção dos pacientes domiciliares e cuidadores acerca das ações para a prevenção de LP na Estratégia Saúde da Família. Desta, emergiram duas subcategorias: a) percepção de pacientes domiciliares e cuidadores acerca da LP; b) medidas de prevenção para LP realizadas por pacientes e cuidadores. Conclusão: os participantes da pesquisa

\footnotetext{
${ }^{1}$ Enfermeira. Pós-Graduanda em Enfermagem em Estomaterapia pela Universidade Regional do Cariri. Enfermeira Assitencial do Ambulatório de Enfermagem em Estomaterapia da Universidade Regional do Cariri. Crato, Ceará, Brasil. E-mail: tatyelle bc@hotmail.com ORCID ID: https://orcid.org/0000-0001-6515-7963

${ }^{2}$ Enfermeiro. Estomaterapeuta TISOBEST. Doutor em Farmacologia. Docente Adjunto da Universidade Regional do Cariri. Crato, Ceará, Brasil. E-mail: rafael.sampaio@urca.br ORCID ID: https://orcid.org/0000-0003-14379421 Autor principal - Endereço para correspondência: Rua Cel. Antônio Luis, 1161 - 63105-000 - Pimenta Crato/CE, Brasil.

${ }^{3}$ Enfermeira. Universidade Regional do Cariri. Crato, Ceará, Brasil. E-mail: brendalunabelem@gmail.com ORCID ID: https://orcid.org/0000-0002-1274-4301

${ }^{4}$ Enfermeira. Mestrando em Enfermagem pela Universidade Regional do Cariri. Enfermeira Assitencial do Ambulatório de Enfermagem em Estomaterapia da Universidade Regional do Cariri. Crato, Ceará, Brasil. E-mail: neyzemartins4@gmail.com ORCID ID: https://orcid.org/0000-0001-8371-3150

${ }^{5}$ Enfermeira. Doutora em Bioquimica Toxicológica. Docente Adjunta da Universidade Regional do Cariri. Crato, Ceará, Brasil. E-mail: cleidecorreia27@hotmail.com ORCID ID: https://orcid.org/0000-0001-8135-449X
} meio, desde que a publicação original seja corretamente citada. 
detém o conhecimento acerca da LP baseado nas experiências diárias, desenvolvendo efetivamente as ações para a prevenção das lesões.

Descritores: Lesão por Pressão; Atenção Primária à Saúde; Cuidadores.

\section{ABSTRACT}

Objective: to know the actions performed at home for the prevention of pressure injuries in the Family Health Strategy. Method: descriptive and qualitative research directed at the population at risk for the development of pressure injury (PI) in the city of Crato, Ceará, Brazil. Data collection was performed through the Braden scale and semi-structured interview, from March to May 2018. The ethical aspects involved in the research were respected. Results: the results obtained were divided into two categories: 1- analysis of the risk of developing PI from the Braden scale; 2- perception of home patients and caregivers about actions for the prevention of $\mathrm{Pl}$ in the Family Health Strategy. From this, two subcategories emerged: a) perception of home patients and caregivers about PI; b) prevention measures for PI performed by patients and caregivers. Conclusion: the research participants have knowledge about PI based on daily experiences, effectively developing actions for injury prevention.

Descriptors: Pressure Ulces; Primary Health Care; Caregivers.

\section{RESUMEN}

Objetivo: conocer las acciones realizadas en el hogar para la prevención de lesion por presión en la Estrategia de Salud de la Familia. Método: investigación descriptiva y cualitativo, dirigida a la población en riesgo de desarrollar lesiones por presión (LP) en la ciudad de Crato, Ceará, Brasil. La recolección de datos se realizó a través de la escala de Braden y la entrevista semiestructurada, de marzo a mayo de 2018. Se respetaron los aspectos éticos involucrados en la investigación. Resultados: los resultados obtenidos se dividieron en dos categorías: 1 - Análisis del riesgo de desarrollar LP a partir de la escala de Braden. 2- Percepción de pacientes domiciliarios y cuidadores sobre acciones para la prevención de LP en la Estrategia de Salud Familiar. De esto, surgieron dos subcategorías: a) Percepción de pacientes domiciliarios y cuidadores sobre las lesiones por presión; b) Medidas de prevención para LP realizadas por pacientes y cuidadores. Conclusión: los participantes de la investigación tienen conocimientos sobre $L P$ basados en experiencias diarias, desarrollando acciones para la prevención de lesiones.

Descriptores: Úlcera por Presión; Estrategia de Salud Familiar; Cuidadores.

\section{INTRODUÇÃO}

O atual cenário populacional
brasileiro se construiu dentre
transições epidemiológicas e
demográficas, associadas ao processo
de envelhecimento populacional, com
modificações nos padrões de morte,

morbidade e invalidez. 0 resultado deste processo resultou em maior número de indivíduos com necessidade de cuidados domiciliares ${ }^{1}$.

No Brasil, houve um crescimento da prevalência de pacientes acompanhados pelo Serviço 
de Atenção Domiciliar (SAD) ${ }^{2}$ com lesão por pressão (LP). E em estudos realizados no Canadá 3 e Japão ${ }^{4}$, além da prevalência dessas lesões, há preocupação quanto ao cuidado familiar e doméstico, pois nem sempre ocorrem de modo a favorecer a melhora das LP.

$\mathrm{Na}$ literatura nacional, os estudos são mais limitados às descrições sobre LP e evolução da lesão, do que aos impactos causados. Porém, estudo local em uma cidade paulista, ressalta a importância dessas repercussões, ao observar que $70,2 \%$ dos pacientes domiciliares apresentaram risco de desenvolver LP, com possibilidade de agravamento, já que boa parte desses indivíduos não possuem ou não são assistidos por cuidadores esclarecidos e/ou com disposição/disponibilidade 5 .

Dentro dessa perspectiva insere-se os cuidados domiciliares como uma estratégia importante para a prevenção das LP. Estes cuidados são considerados peças chave para a continuidade da assistência, instituída e fortalecida a partir das orientações ofertadas pelo serviço de saúde. Assim, a participação do cuidador, seja ele familiar ou profissional contratado, poderá ser uma forma de efetivação das ações preventivas ${ }^{6,7}$.

Diante disso, essa pesquisa surge a partir da necessidade de conhecer as estratégias preventivas para as LP no âmbito da atenção primária, direcionado ao cuidado domiciliar. Face ao exposto, a presente investigação teve como objetivo, conhecer as ações realizadas no domicílio para a prevenção de LP na Estratégia Saúde da Família (ESF).

\section{MÉTODO}

Trata-se de estudo descritivo e qualitativo, realizado em uma ESF no Nordeste brasileiro, na região do Cariri, no município de Crato-CE, entre os meses de março e maio de 2018. Crato é um município brasileiro do estado do Ceará, com área territorial de 1.176,467 km². Localizase no sopé da Chapada do Araripe no extremo-sul do estado e na Microrregião do Cariri. Em 2018, possuía 131.372 habitantes e densidade demográfica $103,21 \mathrm{hab} / \mathrm{km}^{2}$. É uma das cidades mais importantes e antigas do Ceará, situando-se atualmente como a sexta cidade mais populosa e a terceira mais desenvolvida com o sétimo maior PIB do Estado ${ }^{8}$. 
A coleta de dados foi realizada com os pacientes domiciliares e seus cuidadores, familiares ou profissionais contratados, que residiam ou cuidavam dos pacientes residentes nas áreas adscritas pelas fichas do eSUS Atenção Básica (e-SUS AB) deste mesmo município. 0 e-SUS $A B$ é uma estratégia do Departamento de Atenção Básica do Ministério da Saúde (MS) para reestruturar o registro de informações das ações realizadas na Atenção Básica $(A B)$ em nível nacional'9. Quando na impossibilidade do paciente domiciliar responder ao instrumento de coleta de dados, devido a incapacidade de estabelecer comunicação verbal, foi entrevistado o cuidador.

Foram inclusos participantes maiores de 18 anos, acamados ou cadeirantes, que não haviam desenvolvido LP durante o período da pesquisa. Participantes que já possuíam LP ou deambulavam com dificuldade foram excluídos. Assim foram incluídos no estudo 11 participantes, dentre esses, quatro pacientes ativos iminentes ao risco de desenvolver LP por estarem com a mobilidade física prejudicada e sete cuidadores domiciliares.
Para a seleção inicial dessa amostra, considerou-se os 2808 pacientes da unidade de saúde que residiam nas áreas de cobertura de três Agentes Comunitários de Saúde (ACS) atuantes no serviço. Posteriormente, a enfermeira da unidade, juntamente com os ACS, facilitaram acesso aos participantes, além de acompanhar toda visita domiciliar.

Após a seleção dos participantes, 0 pesquisador juntamente com a ACS da devida micro área, realizaram visita domiciliar, apresentando-se e explicando sobre a pesquisa. Após a aceitação, solicitou-se a assinatura do Termo de Consentimento Livre e Esclarecido (TCLE).

A coleta de dados ocorreu em duas etapas, a primeira por meio da aplicação da escala de risco de Braden aos pacientes domiciliares, com preenchimento dos scores, a fim de classificar o risco para 0 desenvolvimento de LP dos pacientes alvo da pesquisa. Essa escala avalia seis categorias diferentes: 1 percepção sensorial; 2 - umidade; 3 atividade; 4 - mobilidade; 5 nutrição; 6 - fricção e cisalhamento. Ela funciona de forma crescente, 
quanto maior o número, melhor o resultado para risco diminuído (risco muito alto, 06 a 09; risco alto, 10 a 12; risco moderado, 13 a 14; risco baixo, 15 a 18; sem risco, 19 a 23) ${ }^{10}$. Já a segunda, foi realizada entrevista semiestruturada com as seguintes questões: 1) Você sabe o que é úlcera por pressão, lesão por pressão ou "escara"?; 2) Como você realiza a prevenção de feridas?; 3) Já recebeu alguma orientação dos profissionais de saúde sobre a prevenção ou os cuidados com as feridas?". Para a coleta, um pesquisador devidamente capacitado foi a campo, gravando os relatos em áudio, que posteriormente foram transcritos na íntegra e codificados por uma letra do alfabeto para cada paciente, de acordo com a ordem de realização da entrevista.

A análise dos dados foi realizada por meio da técnica de Análise de Conteúdo de Bardin, seguindo as etapas de pré-análise, exploração do material e tratamento dos resultados, inferência e interpretação. Entende-se por análise de conteúdo um conjunto de instrumentos metodológicos que visa analisar diferentes formas de comunicação, verbal ou não verbal, obtida em entrevistas ou observação direta, cujo material, extraído após análise minuciosa e exaustiva, é classificado/separado em temas ou categorias ${ }^{11}$.

A participação no estudo se deu de forma voluntária e com base na Resolução 466/2012 do Conselho Nacional de Saúde (CNS), tendo aprovação do Comitê de Ética e Pesquisa com Seres Humanos da Universidade Regional do Cariri (URCA), obtendo o parecer de $\mathrm{N}^{\circ}$ 2.639.729

e

CAAE:

82448318.5.0000.5055.

\section{RESULTADOS E DISCUSSÃO}

Participaram 11 usuários, destes, quatro participantes eram pacientes com capacidade de estabelecer a comunicação verbal, um do sexo masculino e três do sexo feminino, e sete cuidadores, todos do sexo feminino. Os participantes da pesquisa estavam na faixa etária de 57 a 93 anos, predominando os idosos.

Os resultados foram divididos em duas categorias: "Análise do risco de desenvolvimento de LP" e "Percepção dos pacientes domiciliares e cuidadores acerca das ações para a prevenção de LP na Estratégia Saúde 
da Família”. Desta última categoria, emergiram duas subcategorias: a) percepção de pacientes domiciliares e cuidadores acerca da LP; b) medidas de prevenção para LP realizadas por pacientes e cuidadores.

\section{Análise do risco de desenvolvimento} de LP

Ao aplicar a escala de risco de Braden, o somatório dos scores revelou que o maior quantitativo de participantes encontrava-se sem risco $(n=5 ; 46 \%)$ para o desenvolvimento das lesões, seguido do baixo risco $(\mathrm{n}=$ 4; $36 \%$ ) para o desenvolvimento e por fim, o risco moderado $(n=2 ; 18 \%)$.

Assim como observado no presente estudo, pesquisa revela que $80 \%$ dos pacientes que desenvolvem as lesões, são do gênero feminino e apenas $20 \%$ do gênero masculino. E quanto a faixa etária, mais de $60 \%$ da população é constituída por idosos ${ }^{12}$.

Quanto a avaliação para o risco de desenvolver LP, obteve-se sem risco e baixo risco como as classificações mais frequentes. Corroborando com este resultado, pesquisa realizada no município de Terezina (PI), demonstrou que $50 \%$ dos 32 pacientes acamados assistidos pela
$A B$, foram classificados com baixo risco para desenvolver LP e nenhum categorizado com risco muito alto. Contudo, a percentagem de $18,8 \%$ de entrevistados que alcançaram escore de Braden menor ou igual a 18 é significativa, indicando risco para desenvolvimento da lesão ${ }^{13}$.

Nessa perspectiva, o processo de avaliação de risco para o desenvolvimento de LP é essencial dentro das propostas de prevenção, uma vez que pretende detectar precocemente o potencial paciente para o desenvolvimento de tais lesões. Nessa perspectiva, a equipe de enfermagem deverá implementar medidas especificas, tais como as intervenções preventivas e avaliação sistemática com instrumentos padronizados ${ }^{14,15}$.

MS recomenda para os pacientes com baixo risco, as seguintes medidas preventivas: cronograma de mudança de posicionamento, otimização da mobilização, proteção do calcanhar, controle de umidade, nutrição, fricção e cisalhamento e uso de superfícies de redistribuição de pressão. Para o risco moderado: todas as medidas para os pacientes de baixo risco, acrescentando 0 
posicionamento em $30^{\circ}$. Para o alto risco são recomendadas as mesmas medidas ao risco moderado, incluindo o uso de almofadas de espuma para facilitar a lateralização. Para pacientes que apresentam risco severo, todos os passos mencionados são recomendados e também o uso de superfícies de apoio dinâmico com pouca perda de $a r^{12}$. Já quando a pontuação acumulativa de risco é classificada como sem risco, existem evidências que recomendem a reavaliação com a escala a cada 48 horas ${ }^{16}$.

Percepção dos pacientes domiciliares e cuidadores acerca das ações para a prevenção de LP

A) Percepção de pacientes domiciliares e cuidadores acerca da LP

Essa subcategoria apresenta a percepção de participantes ou cuidadores acerca da LP, de acordo com o conhecimento prévio, levando a compreensão de que as informações apreciadas nos relatos foram construídas a partir de saberes empíricos.

Em um primeiro momento, foi questionado aos participantes qual o entendimento sobre a definição de
LP, observando incertezas dos entrevistados pelo termo científico, porém após mencionar o termo mais conhecido popularmente "escara", e explicar o seu aspecto de manifestação no corpo, $72,7 \%(n=8)$ dos entrevistados definiram LP de forma eficaz, alguns de forma mais elaborada, fundamentando suas respostas em experiências, e outros mais superficialmente.

Sim. É assim, devido a pessoa ficar muito tempo numa posição, aí cria uma bolha, dessa bolha, ela se desfaz e fica [...] dizem que assim, eu não cheguei a ver, mas dizem que fica na carne viva. (B)

Sei. É as feridas que cria no corpo, por conta da pessoa tá muito tempo acamada. (D)

Sei, é aquele ferimento que dá, devido a pessoa estar muito tempo deitada. (F)

Sim, quando a pessoa passa muito tempo deitada ou sentada. (K)

Embora a maioria dos participantes tenham apresentado elementos cognitivos satisfatórios do entendimento acerca da LP, também a relacionaram com a necessidade de uma higiene corporal bem executada.

Sei [...] são ferimentos, tipo assim, se você não cuidar do idoso e não tiver uma higiene bem profunda, então acontece essas feridas. $(\mathrm{H})$

São ferimentos, provocados muitas vezes por falta de higiene, ou pelo tempo do paciente acamado. (I) 
Quanto a percepção dos participantes da pesquisa acerca da LP e as estratégias preventivas, observou-se que a maioria detinha apropriação dos conceitos chave pertinentes a etiologia das lesões. No entanto, ao serem abordados sobre as intervenções para prevenção, observou-se o desconhecimento das práticas preventivas para o surgimento destas injurias tegumentares. Desta forma, pode-se afirmar que 0 conhecimento apresentado sobre a enfermidade da pele não se traduziu satisfatoriamente em habilidade e atitudes com vista à prevenção.

Similar aos achados obtidos na presente investigação, um estudo realizado na região dos pampas gaúchos, com o intuito de identificar as concepções de saúde dos cuidadores, observou que além da falta de orientações e subsídios prévios para assumir o papel de exercer o cuidado domiciliar, os participantes cuidadores conseguiram identificar $e$ entender os fatores caracterizadores do risco para o desenvolvimento de $\mathrm{LP}^{17}$.

Nesse mesmo estudo, a pouca escolaridade dos cuidadores foi pautada como um dos dados importantes a ser evidenciado, principalmente quando eles relataram receber algumas orientações, mas classifica-las como insuficientes para a continuidade o cuidado. Desse modo, acredita-se que o baixo nível de escolaridade é um fator interveniente para a dificuldade das orientações recebidas ${ }^{17}$.

Corroborando com tal achado, estudo conduzido em ESF de um município do norte do estado do Paraná, demonstrou que os saberes e práticas dos cuidadores familiares referentes à avaliação e classificação da lesão por pressão eram ineficientes ${ }^{18}$. Contudo, pesquisadores afirmam que o desconhecimento dos cuidadores acerca das vivências do cotidiano, por não ter experiência prévia ou o conhecimento adquirido por já terem passado por essa experiência, não era definidor para o cuidado de qualidade pois 0 desenvolvimento das lesões ainda causava impacto quando vista em sua realidade ${ }^{19}$.

B) Medidas de prevenção para LP realizadas por pacientes e cuidadores 
Quando questionado sobre a prevenção das lesões por pressão, $63,6 \%(n=7)$ relataram saber realizar a prevenção das LP, mas a descrição da estratégia utilizada desvelou desconhecimento acerca da prevenção desta condição clínica.

De acordo com as narrativas a seguir, os participantes informam o uso de superfícies de apoio ou permanecer sentado em substituição a mudança de decúbito para prevenção de LP.

A princípio, eu usava aquelas almofadas com água, aí agora eu comprei um colchão que popularmente a gente conhece como quenga de ovo, que tem os furinhos. Mas agora eu comprei um diferente [...]Antes como não tinha, eu ficava virando ela. Aí eu encontrei essa bolsa, comecei com a almofada, colocava só na parte da nádega dela, porque é onde ficava muito vermelho [...]. (B)

O máximo possível ficar sentada, evitar ficar deitada. $(\mathrm{F})$

As principais medidas de prevenção são a avaliação dos pacientes em risco; o manejo do estado nutricional incluindo a hidratação, inspeção e avaliação diária da pele; o manejo da umidade e a redistribuição da pressão. Enfatizando que todos os esforços devem ser feitos para redistribuir a pressão sobre a pele, seja pelo reposicionamento a cada duas horas e/ou pela utilização de superfícies de redistribuição de pressão ${ }^{22}$.

Dentre as visões acerca da prevenção de LP, alguns participantes apresentaram estratégias

consideradas eficazes.

Mudança de decúbito. (K)

Usando o colchão pneumático e botando aquela pomada para prevenir de assaduras. (D)

Através de creme, hidratantes e o asseio normal. (I)

$E$ entre as ações essenciais para a prevenção da LP, tem-se a mobilização e $o$ posicionamento adequado do paciente, cuidados com a pele por meio da hidratação e proteção contra cisalhamento, a utilização de colchões, monitoramento das condições nutricionais e ingestão hídrica, embora não substituam a mudança de decúbito programada para cada duas horas, no mínimo ${ }^{20}$. 0 rodizio de posições é importante, especialmente para evitar a permanência prolongada sob as proeminências ósseas, como a região sacral e glútea ${ }^{22}$.

Nesse contexto, as medidas de prevenção e cuidados com as LP, são fatores primordiais para melhorar a qualidade de vida dos pacientes. Não sendo competência apenas aos 
profissionais de enfermagem, embora sejam a peça chave para promover a saúde e capacitar os cuidadores e familiares ${ }^{23}$.

É importante ressaltar a partir disso, que quando questionados sobre o repasse de orientações referente a prevenção das LP no âmbito da ESF, $100 \%$ dos entrevistados afirmaram não terem recebido informação ou orientação sobre a temática.

Não, de forma nenhuma. (A)

Não. (B-K)

Quando mencionada a participação dos profissionais de enfermagem, por serem os principais facilitadores de informação dentro da unidade primária, os participantes também relataram a ausência de orientações com vistas à prevenção.

Nessa perspectiva, o estudo demonstra que 0 repasse de orientações ainda é deficiente dentro da unidade de saúde estudada. Isso alerta para a maior vigilância não somente na avaliação da pele, mas na capacitação do grupo familiar ${ }^{24}$.

Os profissionais de enfermagem desempenham papel fundamental na prevenção da LP, garantindo a orientação segura para que seja realizada a mobilidade do paciente dentro e fora do leito, evitando fricção e cisalhamento nas superfícies, com alerta para mudança de decúbito de duas em duas horas. Assim, estas ações de prevenção, devem ser difundidas e aplicadas dentro da atenção domiciliar, dando uma autonomia maior a família para o cuidado $^{25}$.

No entanto, essas não devem ser ações especificas apenas da enfermagem, tendo em vista que o trabalho multiprofissional é a base da assistência na atenção primária em saúde. Neste contexto, a educação em saúde integra-se e soma-se ao conceito de promoção da saúde, tanto dentro da unidade de saúde para capacitar os profissionais, como dentro do domicílio, onde os pacientes estão mais próximos dos familiares, inseridos na sensação de conforto e segurança. Assim, potencializa a capacidade de cuidado através da troca de conhecimento, onde um aprende com o outro ${ }^{26}$.

\section{CONCLUSÃO}

Conclui-se que as ações realizadas em domicílio para a prevenção de LP estão fundamentadas no conhecimento prévio dos cuidadores de indivíduos com 
mobilidade física prejudicada, os quais relacionam o surgimento das LP com o atrito e pressão da pele do paciente em superfícies rígidas, fricção ocasionada por vestimentas e higiene corporal inadequada. Quanto às medidas de prevenção, evidenciou a mudança de decúbito dorsal para sentado, o uso de dispositivos como colchões, coxins e almofadas e a higiene adequada. Contudo, não foi citado pelos cuidadores fatores relacionadas a nutrição e alimentação.

Quanto às orientações no âmbito da ESF, sobre a prevenção das LP todos os participantes, tanto pacientes como cuidadores, afirmaram não terem recebido informação ou orientação sobre a temática.

Portanto, esse estudo aponta a necessidade de maior participação dos profissionais e gestores da ESF junto aos pacientes domiciliares e seus cuidadores no que cerne as estratégias de prevenção das LPs. Além de se fazerem necessárias novas pesquisas para suprir a limitação do estudo em identificar quais as falhas da comunicação entre a unidade de saúde e seus usuários domiciliares.

\section{REFERÊNCIAS}

1. Miranda GMD, Mendes ACG, Silva ALA. 0 envelhecimento populacional brasileiro: desafios e consequências sociais atuais $\mathrm{e}$ futuras. Rev Bras Geriatr Gerontol. 2016; 19(3):507-519

2. Ministério da Saúde (BR). Portaria 825, de 25 de abril de 2016: redefine a Atenção Domiciliar no âmbito do Sistema Único de Saúde e atualiza as equipes habilitadas. Brasília: MS; 2016.

3. Woo KY, Sears K, Almost J, Wilson R, Whitehead M, VanDenKerkhof EG. Exploration of pressure ulcer and related skin problems across the spectrum of health care settings in Ontario using administrative data. Int Wound J. 2017; 14(1):24-30.

4. Kamimura $H M$, Lee $H$, Take $A$, Mukaikubo S, Ogata S. Educational Strategies for Preventing Pressure Ulcer in Japan. Bull Sch Health Sci. 2012; 22:(1):7-12.

5. Moro JV, Caliri MHL. Úlcera por pressão e o cuidado em domicílio. Esc Anna Nery. 2016; 20(3): e20160058

6. Moreira PSCR, Souza MMT. Cuidados de enfermagem a lesão por pressão 
- relato de caso. Rev PróUniverSUS. 2018; 09(1):105-110.

7. Gomes MFP, Fracolli LA, Machado BC. Atenção domiciliar do enfermeiro na estratégia saúde da família. Mundo Saúde (Impr.). 2015; 39(4):470-475.

8. IBGE. Instituto Brasileiro de Geografia e Estatística. Indicadores Sociais Municipais: uma análise dos resultados do universo do Censo Demográfico. Rio de Janeiro: IBGE; 2018.

9. Ministério da Saúde (BR). Secretaria de Atenção à Saúde. Departamento de Atenção Básica. Perguntas frequentes da estratégia e-SUS $A B$, Versão Preliminar. Brasília: MS; 2015.

10. Šáteková L, Žiaková K, Zeleníková R. Predictive validity of the Braden Scale, Norton Scale, and Waterlow Scale in the Czech Republic. IJNP. 2017; 23(1):e12499.

11. Bardin L. Análise de conteúdo. São Paulo: Edições 70; 2016.

12. Cabello PAG, Arévalo-Velasco JM. Estimación del coste sanitario de las úlceras por pre sión en pacientes lesionados medulares. Rev Gerenc Polít Salud. 2016; 15(30):60-67.
13. Vieira CPB, Oliveira EWF, Ribeiro MGC, Luz MHBA, Araújo OD. Ações preventivas em úlceras por pressão realizadas por enfermeiros na atenção básica. J res: fundam care Online. 2016; 8(2):4447-4459.

14. Borghardt AT, Prado TN, Bicudo SDS, Castro DS, Bringuente MEO. Úlcera por pressão em pacientes críticos: incidência e fatores associados. Rev Bras Enferm. 2016; 69(3):460-467.

15. Garcia-Días JF, Cabello-Jaime R, Muñoz-Conde $M$, Berguera-Lezaun I, Blanca-Barba F, CarrascoHerrero JM. Validación de las escalas de Braden y EMINA en pacientes de atención domiciliaria incluidos en programa de inmovilizados. Gerokomos. 2015; 26(4):150-156.

16. Gadd MM, Morris SM. Use of the Braden Scale for Pressure Ulcer Risk Assessment in a Community Hospital Setting. J Wound Ostomy Continence Nurs. 2014; 41(6):535538.

17. Nunes JB, Bussanello J, MelloCarpes PB, Cardoso LS, Costa VZ, Deus LML. Concepções de saúde de cuidadores. Cienc Cuid Saude. 2015; 14(4):1462-1470. 
18. Meireles VC, Baldissera VDA. Qualidade da atenção aos idosos: risco de lesão por pressão como condição marcadora. Rev Rene. 2019; 20:e40122

19. Vieira VAS, Santos MDC, Almeida NA, Bernardes MFVG, Mata LRF. Risco de lesão por pressão em idosos com comprometimento na realização de atividades diárias. RECOM. 2018; 8:e2599.

20. Lima ARV, Lima AJL, Gomes HS, Araújo MTF, Holanda SKS, Souza vo, et al. Tecnologia no cuidado ao paciente internado numa unidade de clínica médica: segurança na prevenção de lesão por pressão. Mostra Inter Enferm. 2016; 2(2):1-6.

21. Hartman CW, Solomon J, Palmer JA, Lukas CV. Contextual Facilitators of and Barriers to Nursing Home Pressure Ulcer Prevention. Adv Skin Wound Care. 2016; 29(5):226-238.

22. National Pressure Ulcer Adivisory Panel. European Pressure Ulcer Advisory Panel Pressure Ulcer Prevention and treatment of pressure ulcers: Quick Reference Guide: In haeesler $\mathrm{H}$, ed.
Cambridge Media: Perth (Australia); 2016.

23. Vasconcelos JMB, Caliri MHL. Ações de enfermagem antes e após um protocolo de prevenção de lesões por pressão em terapia intensiva. Esc Anna Nery. 2017; 21(1):1-9.

24. Pereira AL, Araújo HSO, Rocha LM, Martins MA, Silva Neto MG, Ferreira FGL, et al. Ceratose seborreica em idosos de uma região de savana tropical. J Health NPEPS. 2018; 3(1):143-152.

25. Soares CF, Heidemann ITSB. Promoção da saúde e prevenção da lesão por pressão: expectativas do enfermeiro da atenção primária. Texto Contexto Enferm. 2018; 27(2):e1630016

26. Bierhals CCBK, Santos NO, Fengler FL, Raubustt KD, Forbes DA, Paskulin LMG. Necessidades dos cuidadores familiares na atenção domiciliar a idosos. Rev Latino-am Enferm. 2017; 25:e2870. 
Participação dos autores:

- Concepção: Sampaio LRL, Carvalho TB, Sampaio BLL, Fernandes MNM, Oliveira CC.

- Desenvolvimento: Sampaio LRL, Carvalho TB, Sampaio BLL, Fernandes MNM, Oliveira CC.

- Redação e revisão: Sampaio LRL, Carvalho TB, Sampaio BLL, Fernandes MNM, Oliveira CC.

Como citar este artigo: Sampaio LRL, Carvalho TB, Sampaio BLL, Fernandes MNM, Oliveira CC. Prevenção de lesões por pressão: conhecimento e ações de cuidadores e pacientes domiciliares. J Health NPEPS. 2019; 4(2):331-344.

Submissão: $16 / 07 / 2019$

Aceito: $05 / 11 / 2019$

Publicado: 01/12/2019 